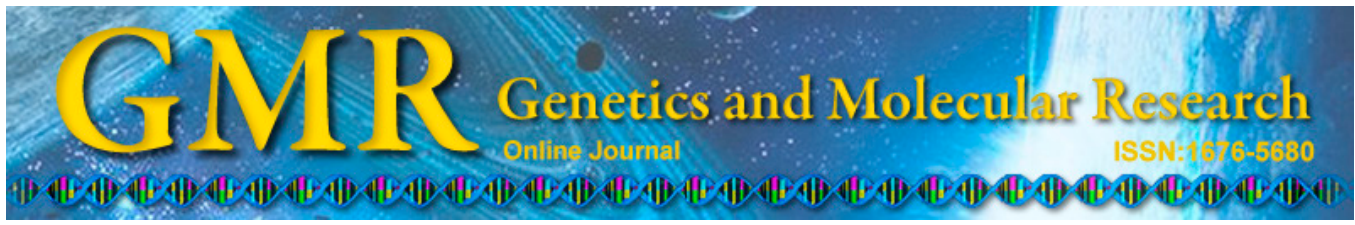

\title{
Expression patterns of melatonin receptors in chicken ovarian follicles affected by monochromatic light
}

\author{
D.Y. Li*, N. Wu*, J.B. Tu*, Y.D. Hu, M.Y. Yang, H.D. Yin, B.L. Chen, \\ H.L. Xu, Y.F. Yao and Q. Zhu
}

Farm Animal Genetic Resources Exploration and Innovation Key Laboratory of Sichuan Province, Sichuan Agricultural University, Ya'an, China

*These authors contributed equally to this study.

Corresponding author: Q. Zhu

E-mail: zhuqingsicau@163.com

Genet. Mol. Res. 14 (3): 10072-10080 (2015)

Received November 27, 2014

Accepted April 29, 2015

Published August 21, 2015

DOI http://dx.doi.org/10.4238/2015.August.21.14

\begin{abstract}
Artificial illumination is an important exogenous factor in the control of many physiological and behavioral processes as well as an important environmental factor in the management of laying hens. Melatonin receptors are members of the $G$ protein-coupled receptor family. The hormone melatonin is secreted primarily by the pineal gland, with highest levels occurring during the dark period of a circadian cycle. The aim of this study was to examine the effect of monochromatic light on chicken egg reproduction and expression of melatonin receptors in chicken ovarian follicles. A total of 552 19-week-old hens were randomly divided into 4 groups with 138 birds in each group. Each group was randomly divided into 3 replicates with 46 birds in each replicate. Feed and water were provided for ad libitum. Light treatments were: control cool white $(400-760 \mathrm{~nm})$, blue $(480$ $\mathrm{nm})$, green $(560 \mathrm{~nm})$, and red $(660 \mathrm{~nm})$. The short wavelength (blue light) group produced a greater total number of eggs at 300 days of age than did the long wavelength (red light) group, and the red light group showed higher melatonin receptor type $1 \mathrm{~A}$ and melatonin receptor
\end{abstract}


type $1 \mathrm{C}$ mRNA and protein expression. These results suggest that the wavelength of light is closely related to chicken egg number at 300 days of age; there is no effect of monochromatic light on melatonin receptor type 1B.

Key words: Egg number; Protein expression; Melatonin receptors; Monochromatic light; Laying hen;

\section{INTRODUCTION}

Artificial illumination is maybe the only source of light provided to birds in modern poultry houses (Rozenboim et al., 1998). Thus, the intensity, duration, and quality of light are important environmental factors (Rozenboim et al., 1998) as light influences both reproductive and productive systems in the domestic fowl. Light is integral to sight, including both visual acuity and color discrimination (Manser, 1996). Vision is the most important sense for birds because good eyesight is essential for safe flight. Birds, compared to humans, have 4 types of color receptors in the eye and have a larger number of nerve connections between the photoreceptors and the brain (Govardovskii and Zueva, 1977). These traits facilitate the birds to perceive not only the human-visible range of light but also the ultraviolet part of the spectrum, as well as aid in the detection of polarized light and magnetic fields (Kelber et al., 2003).

Light cycles allow birds to establish rhythmicity and synchronize many essential functions, including body temperature and the various metabolic processes that facilitate feeding and digestion (Olanrewaju et al., 2006). As an environmental factor, light consists of 3 different aspects that can affect the physical activity of broiler chickens: intensity, color, and photoperiodic regime (Lewis and Morris, 1998). Color and intensity are important in behavioral modifications, while exposure of broilers to darkness is essential for bird health (Olanrewaju et al., 2006). Color is determined by the light wavelength and exerts variable effects on flock performance. Light of different wavelengths show varied stimulated effects on the retina and can result in behavioral changes that can affect growth and development (Xie et al., 2008). Hens in cool white light (CL) produced significantly fewer eggs than those under simulated sunlight light in both laying cycles. Eggs laid by birds housed under blue (BL) or green (GL) light were consistently larger than those under red light (RL) (Pyrzak et al., 1987; Rozenboim et al., 1999). Cao et al. (2008) showed that both BL and GL were more effective for stimulating testosterone secretion and myofiber growth, which led to increasing body growth. These results suggest a possible mechanism for egg reproduction associated with light stimulation.

The growth and development of ovarian follicles in volvea series of complex biochemical and physiological changes, which include hormone receptor expression, steroid biosynthesis, cell proliferation, and differentiation (Liu and Zhang, 2008). Melatonin, which regulates various biological functions through 3 different subtypes of receptors, MTNR1A, MTNR1B, and $M T N R 1 C$, is involved in numerous physiological processes including blood pressure regulation (Doolen et al., 1998), circadian entrainment (Dubocovich et al., 1998), retinal physiology (Bordt et al., 2001), oncogenesis (Bordt et al., 2001), seasonal reproduction (Barrett et al., 1997), ovarian physiology (Clemens et al., 2001), and osteoblast differentiation (Barrett et al., 1997; Roth et al., 1999; Clemens et al., 2001). The MTNR1A and MTNR1B receptor subtypes, encoded by genes on human chromosomes 4 and 11, respectively, are present in humans and other mammals, while an additional melatonin receptor subtype, MTNRIC, has been identified in fish, amphibians, 
and birds. In our previous studies, we found that single-nucleotide polymorphisms (SNPs) in MTNR1A and MTNR1C (Li et al., 2013) affect age at first egg and number of eggs at 300 days of age in Erlang mountainous chickens. In this study, we analyzed the mRNA and protein expression levels of the MTNR1A, MTNR1B, and MTNRIC genes to investigate the effect of monochromatic light on egg production and mRNA and protein expression of melatonin receptors in the ovarian follicles of the Chinese local Erlang mountainous chickens.

\section{MATERIAL AND METHODS}

Materials and methods for bird treatment were based on our previous study (Li et al., 2014). The Animal Welfare Committee of Sichuan Agricultural University approved the animal care protocol used for this experiment (No. DKY-B20110108).

\section{Real-time polymerase chain reaction (PCR)}

The birds were sacrificed by cervical bleeding post anesthesia (thiopental, $90 \mathrm{mg} / \mathrm{kg}$ intravenously). The granulosa layers of preovulatory follicles [SYF, F5, F3, and F1, classified according to Onagbesan et al. (2009)] were separated and snap-frozen in liquid nitrogen. Total RNA isolation, quantification, and synthesis of complementary DNA were conducted according to Yin et al. (2013). The complementary DNA and gene-specific primers for luteinizing hormone/choriogonadotropin receptor (LHCGR) (Liu and Zhang, 2008), MTNR1A (Sundaresan et al., 2009), MTNR1B (Sundaresan et al., 2009), and MTNRIC (Sundaresan et al., 2009) were used to perform reverse transcription-PCR. Gene-specific primers for 9 genes and an endogenous reference gene ( $\beta$-actin) (Wang et al., 2011) were synthesized by Shanghai Sangon Co. Ltd. (Shanghai, China). PCR was performed as described by Yin et al. (2013). An RNA standard curve for each gene was generated based on a modified protocol described by Fronhoffs et al. (2002). All plates were analyzed individually using the software provided with the 7300 Real-Time PCR System (Applied Biosystems, Foster City, CA, USA) using the Auto function. Average gene expression relative to the endogenous control for each sample was calculated using the $2^{-\Delta \Delta \mathrm{Ct}}$ method (Livak and Schmittgen, 2001).

\section{Protein extraction and western blotting}

Ovarian follicle tissues were used for protein isolation using the BSP003 Kit (Sangon Biotech Co., Ltd., Shanghai, China). Protein concentration was measured using the Pierce bicinchoninic acid Protein Assay Kit (Pierce, Rockford, IL, USA) and Varioskan Flash instrument (Thermo Fisher Scientific, Waltham, MA, USA). A total of $30 \mu \mathrm{g}$ protein was resolved by sodium dodecyl sulfate-polyacrylamide gel electrophoresis and then transferred to polyvinylidene fluoride membranes. After blocking with $5 \%$ non-fat milk in $1 \mathrm{X}$ Tris-Buffered saline with Tween 20 buffer for $1 \mathrm{~h}$ at room temperature, membranes were incubated with rabbit anti-chicken gonadotropin and melatonin receptors polyclonal antibodies (Table 1) and rabbit anti-chicken $\beta$-actin polyclonal antibody overnight at $4^{\circ} \mathrm{C}$. The blots were then washed in $1 X$ Tris-Buffered saline with Tween 20 buffer and probed with mouse-anti-rabbit horseradish peroxidase-conjugated $\operatorname{IgG}$ secondary antibody for $1 \mathrm{~h}$ at room temperature. Binding was visualized using enhanced chemi-luminescence (ECL) reagent (ZOMANBIO Inc., Beijing, China) using a ChemiDoc XRS instrument (Bio-Rad, Inc., Hercules, CA, USA). Finally, the 
Quantity One Software (Bio-Rad) was used for densitometry analysis. Information regarding antibodies used in this study is shown in Table 1.

Table 1. Antibody information for all proteins in this study.
\begin{tabular}{lclc}
\hline First antibody & Molecular weight $(\mathrm{kDa})$ & Manufacturers & Dilution ratio \\
\hline Rabbit anti-chicken $\beta$-actin & 42 & Uscnlife & $1: 1000$ \\
Rabbit anti-chicken MTNRIA & 37 & Uscnlife & $1: 100-500$ \\
Rabbit anti-chicken MTNRIB & 45 & Uscnlife & $1: 100-400$ \\
Rabbit anti-chicken MTNRIC & 42 & Uscnlife & $1: 100-500$ \\
\hline
\end{tabular}

All secondary antibodies were the same (mouse-anti-rabbit IgG), diluted 1:2000 in 1X TBST, (Uscnlife Science Inc., Wuhan, China).

\section{Statistical analysis}

Data are reported as least square means \pm SE. All data were analyzed by analysis of variance and the Tukey multiple tests using the SAS 9.3 software (SAS Institute Inc., Cary, $\mathrm{NC}, \mathrm{USA}$ ). A value of $\mathrm{P}<0.05$ was considered to be significantly different.

\section{RESULTS}

\section{Effect of monochromatic light on chicken total number of eggs at 300 days of age}

Effect of monochromatic light on chicken total number of eggs at 300 days of age is presented in Table 2. Birds reared under RL produced fewer eggs compared with those in the BL group (Li et al., 2014).

Table 2. Effect of monochromatic light on chicken total number of eggs at 300 days of age.

\begin{tabular}{lccccc}
\hline Light source & CL & BL & GL & RL & Pooled SE \\
\hline EN300 & $102.84 \pm 1.95^{\text {ab }}$ & $107.16 \pm 1.93^{\mathrm{a}}$ & $104.41 \pm 1.94^{\mathrm{ab}}$ & $100.01 \pm 1.89^{\mathrm{b}}$ & 1.928 \\
\hline
\end{tabular}

Data are reported as least square means \pm SE. Means with different superscript $(a, b, c)$ in the same line for the same item differ significantly $(\mathrm{P}<0.05)$. EN300 = the total number of eggs at 300 days of age (Li et al., 2014).

\section{Effects of monochromatic light on mRNA and protein expression of melatonin receptors}

Reverse transcription-PCR analysis of mRNAs encoding chicken $\beta$-actin, MTNR1A, $M T N R 1 B$, and MTNR1C in ovarian follicles were detected by gel electrophoresis. The gels show the presence of all genes analyzed in this study. Representative western blotting images for proteins in small yellow follicle (SYF) are shown in Figure 1.

The effects of monochromatic light on mRNA and protein expression of melatonin receptors in chicken ovarian follicles are shown in Figure 2 and Table 3, respectively. In the granulosa layers of the preovulatory follicles, the relative abundance of MTNR1A mRNA showed the lowest level in the SYF, which was increased in the granulosa layers of F3. MTNR1A expression under RL was not significantly different between SYF and F5. In SYF, F1, F3, and F5 MTNR1A mRNA expression under RL was significantly higher only when compared to BL. MTNR1A mRNA expression levels in RL were significantly greater than CL in SYF and F3. F5 MTNR1A under RL did not differ from F1 MTNR1A under RL. However, the 
difference between the values for RL compared to BL was greater than the difference between GL compared to BL. MTNRIC also showed the highest mRNA expression level under red light and the highest mRNA expression level in F1. MTNR1C showed the lowest expression in the SYF. F1 MTNR1C under BL was significantly lower compared to F3 MTNR1C under RL and CL.F5 and F3 MTNR1C under RL did not differ from F1 MTNR1C under CL. Monochromatic light did not have a significant effect on the mRNA expression level of MTNR1B. The results of effects of monochromatic light on protein expression of melatonin receptors in chicken ovarian follicles were similar to the mRNA expression pattern (Table 3).

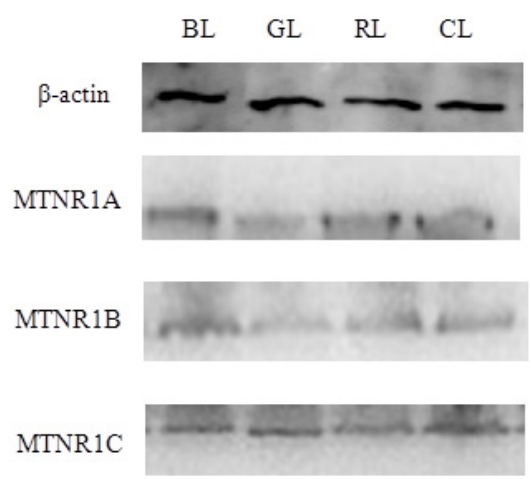

Figure 1. Representative western blotting images.
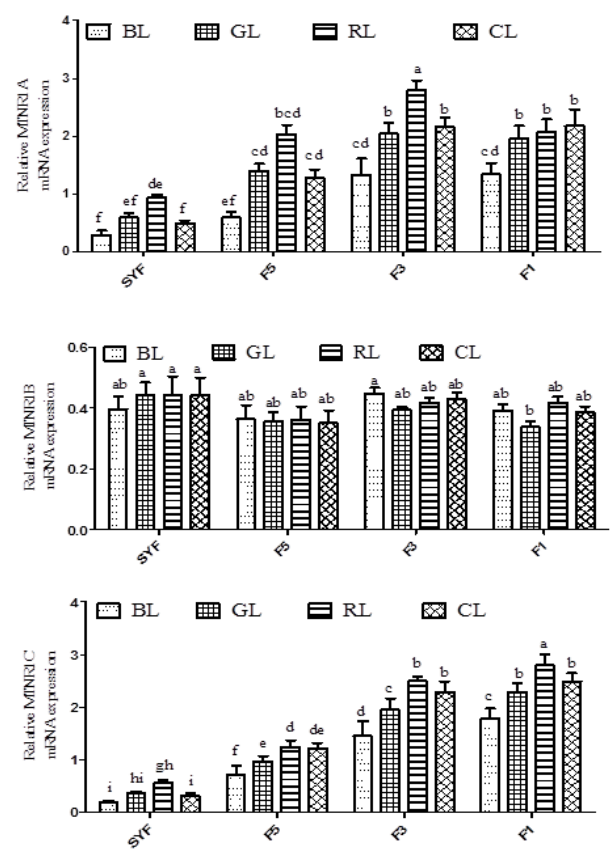

Figure 2. Effects of monochromatic light on mRNA expression of melatonin receptors in chicken ovarian follicles. Values are reported as means $\pm \mathrm{SE}$. Bars with different letters are statistically different $(\mathrm{P}<0.05, \mathrm{~N}=10)$. The mRNA levels are reported as arbitrary units relative to $\beta$-actin mRNA. SYF: small yellow follicle; F5, F3, and F1 $=$ granulosa layers of F5, F3, and F1, respectively. 
Table 3. Effects of monochromatic light on protein expression of melatonin receptors in chicken ovarian follicles.

\begin{tabular}{lllcl}
\hline Follicle & LC & MTNRIA & MTNRIB & MTNRIC \\
\hline SYF & BL & $0.279^{\mathrm{FG}}$ & 0.230 & $0.297^{\mathrm{G}}$ \\
& GL & $0.445^{\mathrm{CDEFG}}$ & 0.220 & $0.127^{\mathrm{HI}}$ \\
& RL & $0.082^{\mathrm{G}}$ & 0.207 & $0.080^{\mathrm{I}}$ \\
F5 & $0.171^{\mathrm{G}}$ & 0.207 & $0.194^{\mathrm{H}}$ \\
& CL & $0.295^{\mathrm{EFG}}$ & 0.234 & $0.361^{\mathrm{FG}}$ \\
& BL & $0.398^{\mathrm{DEFG}}$ & 0.211 & $0.457^{\mathrm{E}}$ \\
& GL & $0.575^{\mathrm{BCDEF}}$ & 0.164 & $0.608^{\mathrm{D}}$ \\
F 3 & $0.398^{\mathrm{DEFG}}$ & 0.207 & $0.431^{\mathrm{EF}}$ \\
& CL & $0.463^{\mathrm{CDEFG}}$ & 0.202 & $0.474^{\mathrm{E}}$ \\
& BL & $0.624^{\mathrm{BCDEF}}$ & 0.215 & $0.655^{\mathrm{CD}}$ \\
& GL & $0.861^{\mathrm{AB}}$ & 0.201 & $0.922^{\mathrm{B}}$ \\
F1 & $0.677^{\mathrm{ABCDE}}$ & 0.201 & $0.722^{\mathrm{C}}$ \\
& CL & $0.703^{\mathrm{ABCD}}$ & 0.203 & $0.735^{\mathrm{C}}$ \\
& BL & $0.812^{\mathrm{ABC}}$ & 0.203 & $0.842^{\mathrm{B}}$ \\
& GL & $1.025^{\mathrm{A}}$ & 0.196 & $1.092^{\mathrm{A}}$ \\
& RL & $0.861^{\mathrm{AB}}$ & 0.209 & $0.875^{\mathrm{B}}$ \\
\hline
\end{tabular}

$\mathrm{LC}=$ light color. Least square means in a column with different letters are significantly $\left({ }^{\mathrm{A}, \mathrm{BP}}<0.05\right)$ different.

\section{DISCUSSION}

Light is the dominant environmental factor regulating melatonin biosynthesis in vertebrates, and regardless of whether a species is diurnal/nocturnal or exhibits crepuscular activity, pineal melatonin levels (difference between nighttime and daytime) are high during the dark phase of a natural or imposed illumination cycle (Reiter, 1991; Arendt, 2002). High level melatonin can increase the plasma level of luteinizing hormone (LH) and follicle-stimulating hormone (FSH) (Karsch, 1987). Low-level LH and FSH can inhibit ovulation (Odell and Swerdloff, 1968). The regulation of gonadotropin secretion is very complex. In birds, there are 2 pivotal neuroendocrine systems regulating the reproductive cycle: the gonadotropin-releasing hormone/gonadotropin system and the vasoactive intestinal polypeptide/prolactin system (Proudman et al., 2006). With respect to biological actions of gonadotropins within hen ovary, FSH binding to granulosa and theca cell membranes has been reported to decrease as the follicle development stage approaches the ovulation time (Etches and Cheng, 1981; Ritzhaupt and Bahr, 1987; Johnson et al., 1996). As in the mammalian receptor, there is considerable evidence that after interaction between LH and the LHCGR, the second messenger signaling mechanisms in the hen ovary includes activation of the adenylyl cyclase/protein kinase A and phospholipase C/phosphatidylinositol systems (Calvo et al., 1981).

The search for molecular markers that influence reproductive traits in chickens has been reported previously (Zhou et al., 2010). The number of eggs at 300 days of age and age at first egg are the most economically important reproductive traits, and much effort has been put into improving these traits (Kang et al., 2011). Our 4 previous studies showed that LHCGR, MTNRIA, MTNRIC, and PRLR SNPs were associated with chicken egg reproduction, but that MTNR1B was not (Zhang et al., 2012; Diyan et al., 2013; Li et al., 2013). We previously (Li et al., 2013) found there are 4 novel polymorphisms [JQ249890:g.384T $>$ C (MTNR1A), JQ249891:g.387T >C (MTNR1A), JQ249894:g.63C > T (MTNR1B), and JQ249896:g.294G>A $(M T N R 1 C)]$ in the melatonin receptor genes. A statistically significant association $(\mathrm{P}<0.01)$ was found between 3 SNPs (MTNRIASNPs and MTNRIC SNP) and reproductive traits (Li et al., 2013). However, MTNR1B had no effect on chicken egg production. mRNA expression analysis revealed no effect of monochromatic light on MTNR1B gene expression. There 
was also no significant difference in MTNR1B protein content among different groups. All mRNA expression, western blot analysis, and SNP data showed that MTNR1B had no effect on chicken egg production.

Overall, we found that birds housed under BL showed the highest egg production and the lowest melatonin receptor mRNA expression level. This may be because of the photo stimulation of retinal photoreceptors, which are sensitive to BL-GL and appear to limit their productive activity in birds (Mobarkey et al., 2010), as well as iodopsin present in relatively large amounts in both chicken and turkey (Crescitelli et al., 1964). Photo stimulation of retinal photoreceptors, which are sensitive to RL, appears to inhibit reproductive activity in birds, and our study showed that birds reared under RL produce fewer eggs than other groups at 300 days of age. According to the results of our study, monochromatic light is associated with egg reproduction traits. The egg production rate of Sichuan native mountainous chickens is relatively low compared to commercial egg type chickens, but this rate can be improved. The mRNA expression levels of melatonin receptors showed that birds housed under BL have a lower level of melatonin receptors (except MTNR1B) expression, suggesting that short wavelength light (BL) may decrease plasma melatonin in birds. Our previous study showed that plasma LH and FSH under BL were higher than under RL, while plasma prolactin under RL was higher than under BL (Li et al., 2014). A low level of melatonin would increase plasma LH and FSH and decrease plasma PRL, as well as promote egg production. We describe one mechanism (Figure 3) of how monochromatic light may affect egg reproduction in chickens.

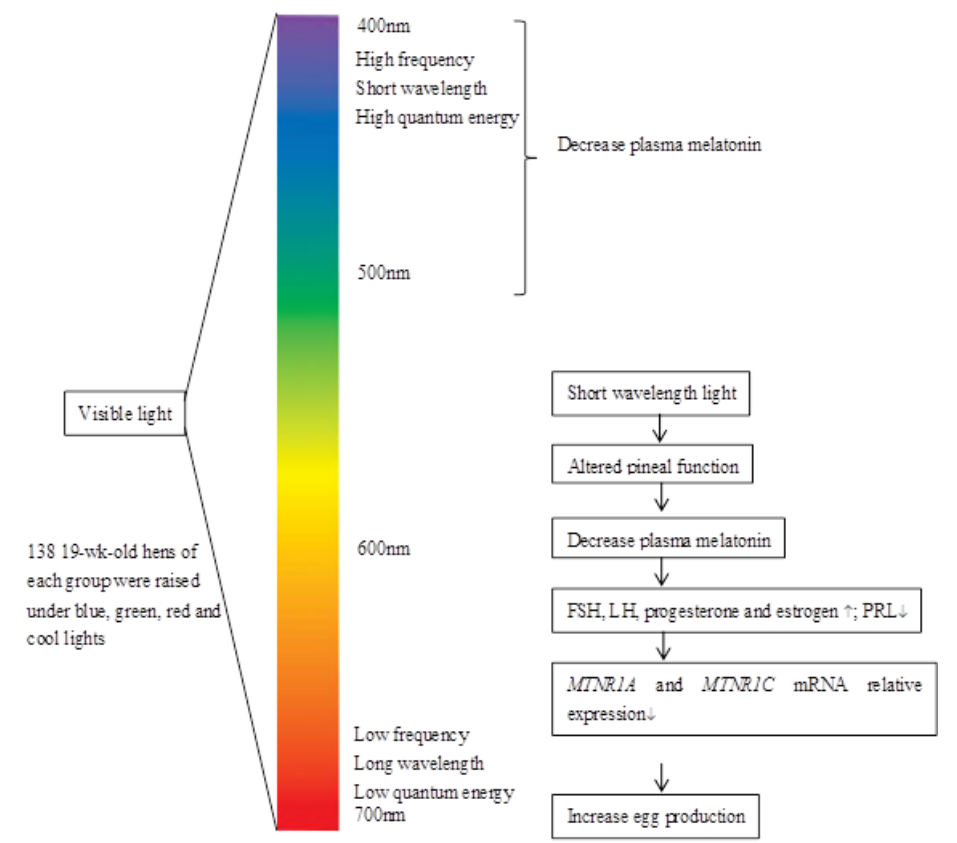

Figure 3. Possible mechanism of how monochromatic light affects egg reproduction in chickens. Birds reared under short wavelength light (BL) have lower plasma melatonin levels. Lower melatonin will increase plasma FSH, $\mathrm{LH}$, progesterone, and estrogen, but will decrease plasma prolactin. Increase in FSH, LH, and progesterone and estrogen will improve egg production. 


\section{CONCLUSIONS}

In the current study, we analyzed the effect of monochromatic light expression of melatonin receptors in chicken ovarian follicles. The results suggested that the wavelength of light was closely related to chicken egg reproductive traits. Short wavelength light (BL) group chickens appeared to have higher egg production, while long wavelength light (RL) group chickens showed greater MTNR1A and MTNRIC mRNA and protein expression level in chicken ovarian follicles. Both relative mRNA expression and SNP analysis suggested that the MTNRIB gene had no effect on chicken egg reproduction.

\section{Conflicts of interest}

The authors declare no conflict of interest

\section{ACKNOWLEDGMENTS}

Research supported by the China Agriculture Research System (\#CARS-41), and the Program from Sichuan Province (\#2011NZ0099-7 and \#2011NZ0073). We thank Yao Zhang and Yan Wang for helping with data collection.

\section{REFERENCES}

Arendt J (2002). Is melatonin a photoperiodic signal in humans? Adv. Exp. Med. Biol. 460: 417-424.

Barrett P, Conway S, Jockers R, Strosberg AD, et al. (1997). Cloning and functional analysis of a polymorphic variant of the ovine Mel la melatonin receptor. Biochim. Biophys. Acta 1356: 299-307.

Bordt SL, McKeon RM, Li PK, Witt-Enderby PA, et al. (2001). N1E-115 mouse neuroblastoma cells express MT melatonin receptors and produce neurites in response to melatonin. Biochim. Biophys. Acta 1499: 257-264.

Calvo F, Wang S and Bahr J (1981). LH-stimulable adenylyl cyclase activity during the ovulatory cycle in granulosa cells of the three largest follicles and the postovulatory follicle of the domestic hen (Gallus domesticus). Biol. Reprod. 25: 805-812.

Cao J, Liu W, Wang Z, Xie D, et al. (2008). Green and blue monochromatic lights promote growth and development of broilers via stimulating testosterone secretion and myofiber growth. J. Appl. Poult. Res. 17: 211-218.

Clemens J, Jarzynka M and Witt-Enderby P (2001). Down-regulation of mt1 melatonin receptors in rat ovary following estrogen exposure. Life Sci. 69: 27-35.

Crescitelli F, Wilson BW and Lilyblade AL (1964). The visual pigments of birds. I. The turkey. Vision Res. 4: 275-280.

Diyan L, Zhang L, Yang MY, Xu HL, et al. (2013). Effect of luteinizing hormone/choriogonadotropin receptor (LHCGR) gene on chicken reproductive traits. Mol. Biol. Rep. 40: 7111-7116.

Doolen S, Krause D, Dubocovich M and Duckles S (1998). Melatonin mediates two distinct responses in vascular smooth muscle. Eur. J. Pharmacol. 345: 67-69.

Dubocovich ML, Yun K, Al-Ghoul WM, Benloucif S, et al. (1998). Selective MT2 melatonin receptor antagonists block melatonin-mediated phase advances of circadian rhythms. FASEB J. 12: 1211-1220.

Etches R and Cheng K (1981). Changes in the plasma concentrations of luteinizing hormone, progesterone, oestradiol and testosterone and in the binding of follicle-stimulating hormone to the theca of follicles during the ovulation cycle of the hen (Gallus domesticus). J. Endocrinol. 91: 11-22.

Fronhoffs S, Totzke G, Stier S, Wernert N, et al. (2002). A method for the rapid construction of cRNA standard curves in quantitative real-time reverse transcription polymerase chain reaction. Mol. Cell. Probes 16: 99-110.

Govardovskii VI and Zueva LV (1977). Visual pigments of chicken and pigeon. Vision Res. 17: 537-543.

Johnson A, Bridgham J and Wagner B (1996). Characterization of a chicken luteinizing hormone receptor (cLH-R) complementary deoxyribonucleic acid, and expression of cLH-R messenger ribonucleic acid in the ovary. Biol. Reprod. 55: 304-309. 
Kang L, Zhang N, Zhang Y, Yan H, et al. (2011). Molecular characterization and identification of a novel polymorphism of $200 \mathrm{bp}$ indel associated with age at first egg of the promoter region in chicken follicle-stimulating hormone receptor (FSHR) gene. Mol. Biol. Rep. 39: 2967-2973.

Karsch FJ (1987). Central actions of ovarian steroids in the feedback regulation of pulsatile secretion of luteinizing hormone. Annu. Rev. Physiol. 49: 365-382.

Kelber A, Vorobyev M and Osorio D (2003). Animal colour vision - behavioural tests and physiological concepts. Biol. Rev. Camb. Philos. Soc. 78: 81-118.

Lewis P and Morris T (1998). Responses of domestic poultry to various light sources. World Poult. Sci. J. 54: 7-25.

Li D, Zhang L, Yang M, Yin H, et al. (2014). The effect of monochromatic LED light on reproductive traits of laying hens. J. Appl. Poult. Res. 23: 367-375.

Li DY, Zhang L, Smith DG, Xu HL, et al. (2013). Genetic effects of melatonin receptor genes on chicken reproductive traits. Czech. J. Anim. Sci. 58: 58-64.

Liu HY and Zhang CQ (2008). Effects of daidzein on messenger ribonucleic Acid expression of gonadotropin receptors in chicken ovarian follicles. Poult. Sci. 87: 541-545.

Livak KJ and Schmittgen TD (2001). Analysis of relative gene expression data using real-time quantitative PCR and the $2^{\Delta \mathrm{ACt}}$ method. Methods 25: 402-408.

Manser CE (1996). Effects of lighting on the welfare of domestic poultry: a review. Anim. Welfare 5: 341-360.

Mobarkey N, Avital N, Heiblum R and Rozenboim I (2010). The role of retinal and extra-retinal photostimulation in reproductive activity in broiler breeder hens. Domest. Anim. Endocrin. 38: 235-243.

Odell WD and Swerdloff RS (1968). Progestogen-induced luteinizing and follicle-stimulating hormone surge in postmenopausal women: a simulated ovulatory peak. Proc. Natl. Acad. Sci. U. S. A. 61: 529-536.

Olanrewaju H, Thaxton J, Dozier W, Purswe J, et al. (2006). A review of lighting programs for broiler production. World Poult. Sci. J. 5: 301-308.

Onagbesan O, Bruggeman V and Decuypere E (2009). Intra-ovarian growth factors regulating ovarian function in avian species: a review. Anim. Reprod. Sci. 111: 121-140.

Proudman JA, Scanes CG, Johannsen SA, Berghman LR, et al. (2006). Comparison of the ability of the three endogenous GnRHs to stimulate release of follicle-stimulating hormone and luteinizing hormone in chickens. Domest. Anim. Endocrin. 31: 141-153.

Pyrzak R, Snapir N, Goodman G and Perek M (1987). The effect of light wavelength on the production and quality of eggs of the domestic hen. Theriogenology 28: 947-960.

Reiter RJ (1991). Pineal melatonin: cell biology of its synthesis and of its physiological interactions. Endocr. Rev. 12: 151-180.

Ritzhaupt LK and Bahr JM (1987). A decrease in FSH receptors of granulosa cells during follicular maturation in the domestic hen. J. Endocrinol. 115: 303-310.

Roth JA, Kim BG, Lin WL and Cho MI (1999). Melatonin promotes osteoblast differentiation and bone formation. J. Biol. Chem. 274: 22041-22047.

Rozenboim I, Zilberman E and Gvaryahu G (1998). New monochromatic light source for laying hens. Poult. Sci. 77 : 1695-1698.

Rozenboim I, Biran I, Uni Z, Robinzon B, et al. (1999). The effect of monochromatic light on broiler growth and development. Poult. Sci. 78: 135-138.

Sundaresan NR, Marcus Leo MD, Subramani J, Anish D, et al. (2009). Expression analysis of melatonin receptor subtypes in the ovary of domestic chicken. Vet. Res. Commun. 33: 49-56.

Wang Y, Zhu Q, Zhao XL, Yao YG, et al. (2011). Age-related expression profile of the SLC27A1 gene in chicken tissues. Mol. Biol. Rep. 38: 5139-5145.

Xie D, Wang Z, Cao J, Dong Y, et al. (2008). Effects of monochromatic light on proliferation response of splencyte in broilers. Anat. Histol. Embryol. 37: 332-337.

Yin HD, Wang Y, Zhang ZC, Liu YP, et al. (2013). Characterization of the expression profile and genetic polymorphism of the cellular retinol-binding protein (CRBP IV) gene in Erlang mountainous chickens. Int. J. Mol. Sci. 14: 4432-4443.

Zhang L, Li D, Liu Y, Wang Y, et al. (2012). Genetic effect of the prolactin receptor gene on egg production traits in chickens. Genet. Mol. Res. 11: 4307-4315.

Zhou M, Du Y, Nie Q, Liang Y, et al. (2010). Associations between polymorphisms in the chicken VIP gene, egg production and broody traits. Br. Poult. Sci. 51: 195-203. 